\title{
e-Phaïstos
}

e-Phaïstos

Revue d'histoire des techniques / Journal of the history

of technology

IV-2 | 2015

Patrimoines de l'eau

\section{Antoine Picon, Smart cities. Théorie et critique d'un idéal auto-réalisateur}

Smart cities: Theory and criticism of a self-fulfilling ideal

Bénédicte Rolland-Villemot

\section{OpenEdition}

Journals

Édition électronique

URL : http://journals.openedition.org/ephaistos/799

DOI : 10.4000/ephaistos.799

ISSN : 2552-0741

Éditeur

IHMC - Institut d'histoire moderne et contemporaine (UMR 8066)

Édition imprimée

Date de publication : 1 octobre 2015

Pagination : $94-97$

ISSN : 2262-7340

Référence électronique

Bénédicte Rolland-Villemot, «Antoine Picon, Smart cities. Théorie et critique d'un idéal auto-

réalisateur », e-Phaïstos [En ligne], IV-2 | 2015, mis en ligne le 30 novembre 2016, consulté le 23

septembre 2020. URL : http://journals.openedition.org/ephaistos/799 ; DOI : https://doi.org/10.4000/ ephaistos.799 


\title{
Antoine Picon, Smart cities. Théorie et critique d'un idéal auto-réalisateur (collection Actualités, Paris, éditions B2, 2013, 120 pages)
}

\author{
Bénédicte Rolland-Villemot \\ Conservateur en chef du patrimoine \\ Doctorante, équipe d'Histoire des \\ Techniques / IHMC \\ CNRS - Université Paris 1 Panthéon- \\ Sorbonne
}

[NDLR : le tirage de cet ouvrage est épuisé mais téléchargeable gratuitement sur le site de l'éditeur: http://editions-b2.com/les-livres/6-smart-cities.htmll

\section{Introduction}

Antoine Picon est ingénieur, architecte et docteur en histoire. Directeur de recherche à l'École nationale des Ponts et Chaussées (laboratoire LATTS) et professeur à l'Université Harvard, il enseigne l'histoire de l'architecture et des techniques. Il est connu notamment pour ses travaux sur les ingénieurs de l'époque moderne ${ }^{1}$. Il est aussi connu pour ses interrogations sur les évolutions des villes, notamment en liens avec les technologies de l'information. En 1997 avec Marc Desportes dans De l'espace au territoire ${ }^{2}$ consacré à l'aménagement des villes en France, il avait étudié ce concept de " ville des réseaux ». Ce premier ouvrage avait été suivi par La ville territoire des cyborgs ${ }^{3}$. Ce nouvel ouvrage paru aux éditions B2 prolonge ce questionnement.

Les éditions B2 ont comme projet éditorial de construire un " cabinet de curiosité [...] architectural arpentant dans le temps et l'espace de Los Angeles à Vladivostok et de l'an mil à nos jours, une infinité d'espèces, d'espaces et d'hétérotopies 4 baroques ». Cette collection s'articule autour de sept thèmes : design, actualités, territoires, société, contre-culture, patrimoine et fac-similé. Ce petit ouvrage très incisif et éclairant paraît dans le thème « actualités ».

L'interrogation porte sur la puissance de l'imaginaire des réseaux. Comment cet imaginaire renvoie-t-il à la fois aux idéaux de domination et à ceux de d'émancipation individuelle et collective ? Pour questionner cette ambivalence, A. Picon s'est intéressé à " la ville des réseaux 》 autour de deux axes. Le premier analyse la tension qui existe entre passé et présent, où il considère la ville haussmannienne comme la genèse de la ville contemporaine des réseaux. Le second porte sur le rapport entre le " contrôle et la volonté de liberté » et prend pour exemple la « ville intelligente » (Smart city). L'auteur s'inquiète de l'absence de réflexion critique qui accompagne le bouleversement de notre environnement urbain. 


\section{Définir la smart city}

L'expression « ville intelligente », traduction de l'anglais smart city, s'emploie pour une ville qui développe les technologies de l'information et de la communication (TIC) pour « améliorer » la qualité des services urbains ou encore réduire ses coûts. Ce concept émergent désigne un type de développement urbain apte à répondre à l'évolution ou à l'émergence des besoins des institutions, des entreprises et des citoyens, tant sur le plan économique, social, qu'environnemental. La gestion du trafic ou la limitation de la consommation électrique entrent par exemple dans ce cadre. Ainsi, une ville intelligente devrait être capable de gérer des infrastructures communicantes entre elles, adaptables, durables et plus efficaces, automatisées pour améliorer la qualité de vie des citoyens, dans le respect de l'environnement.

Ce n'est cependant qu'en 2005 que le terme de smart city a fait son apparition, utilisé par Bill Clinton à l'occasion d'un défi lancé par sa fondation au géant du numérique Cisco. L'ancien président des Etats-Unis avait alors appelé l'entreprise à développer des plans de décongestion des villes, à commencer par San Francisco, Séoul et Amsterdam afin de diminuer les émissions de $\mathrm{CO} 2$ et économiser à la fois pour les citoyens et les communautés locales du temps et de l'argent.

En questionnant ce concept, Antoine Picon relève qu'il est à la fois mal défini et néanmoins opératoire puisque de nombreux projets dans le monde s'en réclament. En fait, dans un contexte mondial d'urbanisation massive, la smart city se présente comme la vision idéale d'une ville qui concilierait le développement durable et la qualité de la vie. Mais n'est-ce pas une utopie?

Pour l'auteur, cette smart city est en rupture avec l'urbanisme traditionnel pour qui l'intelligence était extérieure à l'objet. Dans le cas de la smart city, l'intelligence est intrinsèque à la ville grâce à l'embarquement des technologies de l'information et de la communication et à leur symbiose avec les infrastructures physiques liées aux fonctions essentielles de la ville. L'auteur prend comme exemple la ville de Rio au Brésil où un système est prévu pour surveiller la ville en temps réel, et pour agir rapidement en cas de catastrophe: dévier le trafic en cas d'accident, alerter les bonnes personnes si un égout connaît des ruptures de conduites, ou déclencher les sirènes si de fortes inondations menacent. Elle apparaît alors comme un système d'information capable de mesurer et d'enregistrer les activités humaines et les consommations des citadins qui sont identifiés et situés automatiquement. Son but est de gérer de façon efficace et optimale l'adéquation entre l'offre et la demande des ressources rares, de proposer des services innovants. Elle pourrait même prodiguer conseils et assistance.

L'auteur prend l'adjectif intelligent dans son sens habituel qui est une capacité à apprendre, comprendre et raisonner. En analysant le concept de smart city, l'auteur montre qu'il n'est pas réductible aux techniques et nouvelles technologies disponibles pour la gestion de l'espace urbain disponibles. Cette smart city marque l'émergence d'un être composite ou hybride doté d'une certaine forme d'intelligence. Son but est de créer un lien quasi organique entre l'homme et la cité. Dans le transport et la mobilité intelligente, la ville offre aux citadins une gamme variée de solutions de mobilité répondant à l'ensemble de leurs besoins. Elle peut organiser en temps réel la réponse appropriée, informer, alarmer. Elle serait un être dotée de sentiments. Les citoyens développent une attente importante envers les nouvelles technologies et se construisent un imaginaire. Ils souhaitent que leur ville croisse en symbiose tel un être vivant avec les nouvelles technologies.

\section{Quelles évolutions possibles ?}

Mais la smart city n'échappe pas à la vieille règle qui régit les rapports de l'homme et des techniques; celles-ci sont à la fois sources d'émancipation et 
d'aliénation. Cela conduit à une question fondamentale que pose cet ouvrage sur la gouvernance de la ville intelligente. La smart city évoluera-t-elle vers la tentation néo-cybernétique, sera-t-elle le règne des êtres hybrides entre des systèmes technologiques complexes et des acteurs humains ? Ou bien permettra-t-elle l'émergence d'une nouvelle forme de citoyenneté ? L'auteur pense que vont se développer différents niveaux d'interactions entre des infrastructures techniques (adductions d'eau, voies de communication, moyens de transport....), des logiciels, de l'intelligence incorporée, de la gestion par algorithmes et les citadins.

Mais ce modèle possède ses limites en matière d'écologie, de durabilité culturelle, d'économie, et de société. Et en définitive le choix est politique. Le numérique, le cloud, les nouvelles technologies sont très dévoreuses en énergie. Les nouvelles technologies sont fragiles et leur pérennité n'est pas assurée. Elles sont sensibles aux attaques extérieures: la smart city risque d'être vulnérable au vandalisme, aux cyber-attaques et aux aléas techniques et naturels. De plus, si le politique donne une priorité à l'économie de la connaissance par le développement du numérique il sera confronté à des fractures sociales dans la capacité à utiliser ces ressources. Enfin, les intrusions répétées dans la vie privée et sa marchandisation vont susciter des tensions juridiques, voire des conflits internationaux.

Quel sens donner à cette révolution, comment réinventer la relation entre la ville et le territoire qui l'héberge ? L'apport principal de cet ouvrage réside dans ce questionnement qui prend ses distances avec l'angle technique sous lequel il est le plus souvent abordé. L'urbanisation est une réalité. Plus de la moitié de la population mondiale vit dans les villes aujourd'hui et les estimations à horizon 2050 portent ce chiffre à $70 \%$, c'est à dire 6,5 milliards d'individus. Or les villes représentent $75 \%$ de la consommation d'énergie, $80 \%$ de celle des ressources naturelles et seraient responsable de $80 \%$ de l'effet de serre.

Les attentes des citadins (transports, déchets, éclairage, qualité de l'air, niveau de bruit, sécurité, etc.) interviennent dans un contexte de complexité croissante. De plus, ces attentes s'accompagnent d'une exigence de réactivité, n'échappant pas en cela au rythme imposé par les médias. Ces demandes des citadins sont clairement ressenties par les responsables politiques des grandes villes.

D'un point de vue historique, la smart city est le dernier avatar des nombreuses formes d'expressions qui ont existé dans l'histoire de la relation complexe qu'entretiennent les villes et les technologies de l'information et de la communication. Pour appréhender cette smart city dans sa complexité, il faut donc la situer dans la continuité d'une histoire marquée par l'arrivée de l'ordinateur. Dans la France des années 1960, l'informatique urbaine avait en effet été perçue comme un enjeu fondamental de l'administration des grandes villes, pratiquement une expérience de décentralisation avant la loi.

Dès l'arrivée des réseaux techniques urbains au milieu du XIXe siècle, on a cherché à s'informer sur leurs usages et à améliorer leurs fonctionnements et leur rentabilité pour développer des services et répondre aux besoins des habitants. Les premières mesures de consommations localisées pour les réseaux électriques urbains sont mis en place au début du XXe siècle; les débuts des comptages de trafic dans les années 1920 ; la prévision du débit des eaux pluviales par téléphone puis par les radars ; l'utilisation des images de caméras pour la gestion du trafic (Bison futé en France). Autrement dit, l'« intelligence » a pénétré depuis longtemps au cœur de la gestion urbaine. L'automatisation se généralise dans les transports avec des métros sans conducteur, des locations automatiques de vélos et de voitures, la location de VTC par smartphone. L'« intelligence » est à déjà l'œuvre dans de multiples services à la maison ou dans l'espace urbain. Mais qu'en est-il l'intégration de l'intelligence régulatrice de ces services, vrai marqueur de la smart city, telle qu'elle a été définie?

Sur ce point, un faisceau de technologies dont 
l'émergence est récente - smart grids (réseaux intelligents), données de masse (big data), données ouvertes (open data) notamment - ont créé une opportunité dont se sont saisis les grands industriels du numérique et les responsables politiques locaux. La ville de Rio s'est ainsi dotée d'un système de gestion des crises liées aux inondations avec un centre de crise. Plus récemment ce type de technologie (détection, analyse étayée par des informations de différentes sources, scénarios d'évolution, organisation de la réaction, information des niveaux de décision, synthèse graphique et suivi vidéo de la situation en temps réel, etc.) a été étendu à la sécurité, avec la prévision des délits.

Cette genèse pose la question de l'évolution des villes intelligentes : « comment faire vieillir les villes intelligentes ? ». En effet, étant donné le développement sans précédent des systèmes de recueil de données, il s'agit de savoir si les conurbations mondiales parviendront à dépasser les tensions dialectiques créées entre les nécessités d'une surveillance automatique permanente et la liberté nomade souhaitée par les « habitants-cyborgs » (sic) qui veulent inventer un nouveau métabolisme.

En effet, sans même évoquer le risque de l'avènement d'une société totalitaire du type de celle décrite dans 1984, entièrement sous contrôle du numérique, comment ne pas s'interroger sur la question de la protection des données personnelles, quand la progression du big data tend à faire disparaître la frontière entre données publiques et privées ? Comment aussi ne pas redouter leur piratage par un hacker, ou un terroriste ? Il existe encore peu de réflexions sur ce sujet comme le souligne l'auteur. Plus encore, en n'abordant les questions que sous l'angle technique, n'existe-t-il pas un risque de dépeupler l'" agora », la place publique, de désertifier la vie collective au profit d'une vie individuelle connectée pour résoudre les problèmes de mobilité dans l'immédiateté ?

Alors comment «faire vieillir les smart city »? Antoine Picon n'apporte pas de réponses définitives, mais il a le mérite de poser la question et de fournir des éléments pour alimenter la réflexion dans un petit essai, qui vise à prendre de la hauteur sur ces questions complexes.

${ }^{1}$ PICON Antoine, L'invention de l'ingénieur moderne L'Ecole des Ponts et Chaussées 1747-1851, Paris, Presses de l'Ecole Nationale des Ponts et Chaussées, 1992, 767 p. et PICON Antoine, Les Saint-simoniens Raison, imaginaire et utopie, Paris, Belin, 2002,384 p.

2 DESPORTES Marc et PICON Antoine, De l'espace au territoire L'aménagement en France XVIe-XXe siècles, Paris, Presses de l'Ecole nationale des Ponts et Chaussées, 1997, 222p.

3 PICON Antoine, La ville territoire des cyborgs, Paris, Les éditions de l'imprimeur, 1998, 113 pages.

${ }^{4}$ L'hétérotopie (du grec topos, « lieu », et hétéro, « autre »: « lieu autre ») est un concept forgé par Michel Foucault dans une conférence de 1967 intitulée " Des espaces autres ", publié dans Empan, t.2, $\mathrm{n}^{\circ} 54$, ERES, 2004, accessible sur Cairn: http://www.cairn.info/revue-empan-2004-2-page-12.htm. Il donne comme définition des hétérotopies une localisation physique de l'utopie. Ce sont des espaces concrets qui hébergent l'imaginaire, comme une cabane d'enfant ou un théâtre. 